\title{
Self-inflicted. Deliberate. Death-intentioned. A critical policy analysis of UK Suicide Prevention Policies 2009-2019.
}

\author{
Hazel Marzetti, Alex Oaten, Amy Chandler and Ana Jordan
}

\begin{abstract}
Purpose: With encouragement from the World Health Organisation, national suicide prevention policies have come to be regarded as an essential component of the global effort to reduce suicide. However, despite their global significance, the construction, conceptualisation and proposed provisions offered in suicide prevention policies have, to date, been under researched.
\end{abstract}

Methodological approach: This article addresses this gap, critically analysing eight contemporary UK suicide prevention policy documents in use in all four nations of the United Kingdom between 2009-2019, using Bacchi and Goodwin's post-structural critical policy analysis.

Findings: We argue that across our sample of suicide prevention policies, suicide is constructed as self-inflicted, deliberate and death-intentioned. Consequently, these supposedly neutral definitions of suicide have some significant and problematic effects, often individualising, pathologising, and depoliticising suicide in ways that dislocate suicides from the emotional worlds in which they occur. Accordingly, although suicide prevention policies have the potential to think beyond the boundaries of clinical practice, and consider suicide prevention more holistically, the policies in this sample take a relatively narrow focus, often reducing suicide to a single momentary act and centring death prevention at the expense of considering ways to make individual lives more liveable.

Originality: UK suicide prevention policies have not been subject to critical analysis; this study represents the first attempt to examine the way in which suicide is constructed in UK suicide prevention policy documents.

Key words: suicide prevention; critical policy analysis; UK. 


\section{Background}

Suicide is a major public health concern across the UK, with the latest available figures from the Office for National Statistics and Northern Ireland Statistics and Research Agency reporting rates of suicide in Northern Ireland at 18.6 (per 100,000), compared to 16.1 in Scotland, 12.8 in Wales and 10.3 in England (Northern Ireland Assessmbly Research and Information Service, 2019; Office for National Statistics, 2019). Suicide prevention strategies are considered an essential part of the global response to suicide, with nations that develop prevention policies regarded as demonstrating a serious commitment to reducing deaths by suicide in their country (World Health Organization, 2021). In response to this, and as health and social care is primarily a devolved matter within the UK, all four nations have produced suicide prevention policies; with England and Scotland first producing specific policies in 2002, followed by Northern Ireland and Wales, who published policies in 2006 and 2009 respectively (Mackley, 2019). Such prevention strategies are, however, uniquely positioned to think beyond the boundaries of clinical practice, and to consider suicide prevention more proactively by considering how society can be transformed in ways that make lives more liveable (Yip, 2005).

Suicide research more broadly has been criticised for taking a psycho-centric approach (Rimke, 2016), understanding suicide as the outcome of an individual's mental illness, whilst failing to account for the role of socio-economic and political factors contributing to suicidal thoughts and attempts (Hjelmeland and Knizek, 2017). It has been argued that this individualistic and psychologised focus is in part motivated by a desire to appeal to clinical audiences, where clinicians may lack the power or resources to influence the wider conditions for living in which an individual is situated (Button, 2016; Fitzpatrick, 2021). In contrast, suicide prevention strategies, developed by governments, have the scope to consider immediate crisis intervention alongside more long-term planning to prevent suicide (Yip and Tang, 2021). It is precisely this potential that makes prevention policies an interesting site for critical analysis; however, with notable exceptions (see East, Dorozenko and Martin, 2021 and Fitzpatrick, Hooker and Kerridge, 2015), there is currently little research seeking to understand whether this potential is realised within specific suicide prevention policies.

Aiming to address this gap, this paper uses a critical policy analysis to analyse eight strategic suicide prevention documents in use in the UK between 2009 and 2019. Although suicide and suicide prevention are autonomous concepts, they remain interdependent; without suicide there is no need for suicide prevention (Fitzpatrick, Hooker and Kerridge, 2015); thus a conceptualisation of suicide is a necessary foundation on which suicide prevention policies are built. The ways in which prevention policies construct and define suicide will therefore impact upon their prevention aims and strategies. Consequently, to understand and interrogate contemporary suicide prevention policies, we must begin by examining the ways in which they construct suicide. Throughout the policy documents, we argue that suicide is constructed as self-inflicted, deliberate and death-intentioned. These aspects may not be inherently problematic. However, the ways in which the three defining principles are 
enacted in the policies can result in overly narrow suicide prevention practices that miss opportunities to holistically reconfigure the prevention of suicide as a structural and contextual endeavour.

\section{Methodology}

Our research is guided by Bacchi \& Goodwin's (2016) post-structuralist policy analysis approach, 'what is the problem represented to be?' (WPR). The WPR approach is underpinned by a Foucauldian post-structuralist approach that aims to disrupt the often taken-for-granted notion that societal problems exist independently of the policies that are produced to solve them. Instead, the WPR approach proposes that policies construct the problems that they simultaneously seek to solve and aims to deconstruct this process to allow for critical insights. We therefore begin our analysis by focussing on what the 'problem' is represented to be. We identify and interrogate the assumptions that underlie policy problems in order to understand how they have come into being and how these assumptions actively shape perceptions and possibilities for action (Bacchi and Goodwin, 2010, p.52). WPR provides useful critical and methodological tools for policy analysis and is widely used for these reasons (see, for example, Beutler and Fenech, 2018; Lancaster, Rhodes and Rance, 2020; Lampropoulos and Apostolidis, 2021). In conducting the analysis, we looked at the documents holistically and actively sought to challenge our own readings to recognise unanticipated themes and framings of suicide. Final themes, including those identified within this article, were agreed through dialogue amongst the research team and through close, repeated readings of the policies and the textual evidence we present to support our analysis.

We analysed eight policy documents (seven suicide prevention strategies and one progress report): two documents each from England, Northern Ireland, Scotland and Wales in use between 2009-2019 (see Table 1, below). Whilst each policy represents a discrete production from within a specific national administrative and political context, there remains close policy links between the devolved nations of the UK (and England) and the UK government. The policy documents were coded using NVivo12 qualitative analysis software, with the research team focusing on the ways in which the documents constructed the 'problem' of suicide and how this construction influenced the policies' approach to suicide prevention, alongside other themes. In the penultimate stage of analysis, we undertook a process of descriptive thematic writing (Braun and Clarke, 2006), seeking to understand whether and how themes operated individually and across the data set. In this paper, we focus specifically on how suicide was defined as a 'problem' within the policy documents, and highlight the potential consequences and contradictions stemming from such definitions. All quotes presented in this article are illustrative of broader themes found across documents unless otherwise stated. 
Table 1: Key for 8 suicide prevention strategies, action plans and updates analysed in this paper. England:

ENG2012: HM Government (2012) Preventing Suicide in England: A cross-government outcomes strategy to save lives.

ENG2017: Department of Health (2017) Preventing suicide in England: Third progress report of the crossgovernment outcomes strategy to save lives, Public Health England.

\section{Northern Ireland}

N12012: Department of Health, Social Services and Pubic Safety (2012) Protect Life: A shared vision.

NI2016: Department of Health (2016) Protect Life 2: A draft strategy for suicide prevention in the north of Ireland.

Scotland:

Scot2013: Scottish Government (2013) Suicide Prevention Strategy 2013-16.

Scot2018: Scottish Government (2018) Every life matters : Scotland's suicide prevention action plan.

Wales:

WAL2008: NHS Wales (2008) Talk to me: A national action plan to reduce suicide and self-harm in Wales 2008-13.

WAL2015: Welsh Government (2015) Talk to me 2: Suicide and self harm prevention strategy for Wales 2015-2020.

\section{Findings}

\section{Defining suicide}

The definition of suicide has long been contested, with some arguing that a lack of consistency and precision compromises research quality and the capacity to compare data (Silverman, 2016). However, for others a lack of strict definition accurately captures the fuzzy boundaries between suicidal thoughts, attempts and self-harm, and purposefully resists artificially neat categorisations (Marsh, 2016). Thus, although conceptions of suicide may be perceived as neutral, they are underlined by a range of values and assumptions, which actively construct the subject matter as problems that they then seek to solve (White, 2017). In suicide prevention policies therefore, we argue that the definition of suicidal thoughts, attempts and deaths influences the actions and outcomes that are considered most desirable in the prevention of suicide. In doing so, we consider the consequences, whether intended or not, of the ways in which the policies construct suicide and how this in turn shapes what is considered possible and achievable within policy parameters, privileging certain possible actions whilst reducing or rejecting alternatives. The definitions of suicide 
across the policy documents included in our sample drew upon three unifying principles: firstly, that suicide is self-inflicted; secondly, that it is deliberate; and thirdly, that it is intentioned toward death. Whilst perhaps unintentional, these defining principles can unfortunately result in individualistic and psychologising understandings of suicide, and proposed suicide prevention strategies that prioritise death prevention over the consideration of ways to improve the quality of lives. To make this argument we examine each of the three principles in turn.

\section{Suicide as self-inflicted}

To begin analysing the definition of suicide in the policies, we start with the consistent definition of suicide as self-inflicted. Whilst this could initially be understood as attempting to remove doubt over the possibility of other persons being implicated in an individual's death, doing so can also be understood as foregrounding the agency of the person who dies by suicide in potentially problematic ways:

"Suicide is death resulting from an intentional, self-inflicted act" (see Table 1: Scot2013, p.3; Scot2018, p.20).

When explored further, the seemingly neutral presentation of suicide as an intentional and self-inflicted act by an implied rational agent, can have the effect of stressing the role of the individual and bracketing off the broader societal and political contexts within which such suicides occur. This is not to deny or diminish the ways in which suicide is an individual act in the sense that it is always enacted by an individual person with a unique experience and history. Instead, it is to draw attention to the possible consequence of seeing suicide as solely an individual act. Thus, whilst this portrayal of suicide in a clear-cut and neat manner works to distinguish deaths by suicide from deaths by other methods, it simultaneously risks over-emphasising individual agency, decontextualising and depoliticising suicide (Marsh, 2016).

The policies primarily engaged with structural issues by constructing personal identities and experiences of oppression or marginalisation as risk factors for suicide. In reducing structural violence, marginalisation and stigma to singular risk factors the policies did not appear to holistically consider how these experiences had the potential to affect all aspects of an individual's life. Doing so would include understanding how these experiences pattern individuals' interpersonal relationships, splinter individuals' senses of social belonging, and have wide-ranging impacts on suicidal distress. Instead, across the policies over thirty groups were identified as "at higher risk of suicide", "priority people", or as a group who needed tailored suicide prevention work. Such groups included unemployed people, LGBT people, asylum seekers, and people in touch with the criminal justice system, amongst many others:

"Suicide is the result of highly complex interactions among various risk factors and protective influences which vary from one individual to another. The factors that lead to someone developing a vulnerability to suicidal behaviour (suicidal ideation, suicide planning, suicide attempt) are likely to have their roots in a chain of events and experiences that may have begun years previously, and which, in turn, were shaped 
by broader socioeconomic determinants. The risk factor patterns also vary across age, sex, and geographic location." (NI2016, p.28).

However, despite this acknowledgement, the policies tended not to address this wide range of socio-economic factors in the prevention efforts they suggested. Beyond the rather broad identification of "risk" and the acknowledgement of complexity, there remained a lack of more meaningful engagement with tackling structural contributors, such as wide-ranging inequalities, as methods of suicide prevention. Instead, the policies' proposed preventative actions converged around three key priorities. Firstly, crisis interventions to prevent death by suicide through a range of methods including restricting lethal means; secondly, population surveillance to identify those at risk of suicide; and thirdly, appropriate mental health support; delivered through joined up, cross-sectoral services. Working backwards, if we use these proposed solutions to decipher how the problem is represented in these strategies (Bacchi and Goodwin, 2016), we can see suicide constructed as a moment of mental health crisis, which, if disrupted, may resolve or improve through accessing mental health care:

"People may make a suicide attempt impulsively in direct response to a personal crisis. If lethal means are not easily available to them or if they survive the attempt, suicidal thoughts may pass, or there is time to intervene in other ways or to seek help" (WAL2015, p.17).

Although in the above quote "seek help" is used non-prescriptively, the proposed preventative practices offered by the eight strategies appeared to centre on the provision of mental health care as the primary method of suicide prevention; in doing so, discursively positioning suicide as primarily a mental health problem. As has been argued in other areas of suicide research, the centring of individualistic understandings of suicidal distress, demonstrates a framing of 'the problem' of suicide as a purely psychological problem, distancing suicide from the many socio-economic and structural forces that play a role (Button, 2016; Hjelmeland and Knizek, 2017; Mills, 2018). Suicide therefore becomes individualised, decontextualised and pathologised (Rimke, 2016).

Across the policies there was also a prioritisation of identification and surveillance as methods of suicide prevention. This concentration of energies on short-term crisis prevention methods and mental health support appeared to be premised on the idea of being able to identify individuals 'at risk'. For example, ENG2012 stresses that:

"A range of front-line agencies, including primary and secondary health and social care services, local authorities, the police and Jobcentre Plus, can identify and support (or signpost to support) vulnerable people who may be at risk of suicide" (ENG2012, p.29).

Although superficially this may appear to be an acknowledgment of structural issues such as unemployment impacting on suicide (Stewart, 2019, 2020), the document is completely silent on why a Jobcentre (a service supporting people into employment and administering benefits for people who are unemployed) is a location in which there may be vulnerable and 
suicidal individuals. The Jobcentre is only considered in terms of its ability to act as a location of surveillance and intervention rather than being recognised as site within a nexus of structured inequalities that make lives less liveable (Mills, 2018; Stewart, 2019).

Further to this, it appeared that the responsibility for targeted actions to tackle socioeconomic factors contributing to suicidal distress were pushed outwards from the main body of the suicide prevention strategies into local action plans. Suicide prevention was presented as a shared endeavour, with central government only being one part of the solution. Although this shared approach (with local authorities, Health Boards, and third sector organisations) might allow for suicide prevention to be more tailored to the specific needs of a discrete geographical location, it must also be recognised that the organisations being made responsible for prevention are likely to have significantly less power and resource to make meaningful structural changes. Importantly, despite a clear articulation of the need for a collaborative approach to suicide prevention, there was little written about how this work might be undertaken in practice.

"No one organisation or government department can tackle the issue in isolation. Suicide and self harm prevention needs effective collaboration and joint working across central government departments, between local authorities and health boards, involving the third sector, service users and, of course, clinicians and professionals in a range of settings including schools, workplaces, hospitals and communities" (WAL2015, p.4).

Following on from this, we argue that conceptualisations of suicide as self-inflicted that amplify the agency of the individual in their own death, are mirrored in the policies' proposed solutions. The proposed preventative actions focus on individual, psychological support, and do not explore the potential contributions that ameliorating contextual factors, for example through explicitly working to tackle poverty, discrimination, and marginalisation, could have to suicide prevention. Similarly, Hjelmeland and Knizek (2017) have argued that whilst the role of socioeconomic and political factors in suicidal distress is often acknowledged, and multi-disciplinary approaches to suicide prevention gestured toward (Fitzpatrick, Hooker and Kerridge, 2015); ultimately medicalised and pathologised understandings of suicide and suicide prevention remain privileged.

\section{Suicide as deliberate}

Extending the individualistic conceptualisation of suicide, the policies construct suicide as a deliberate act, pointing towards a suicidal individual who knowingly ends their own life, thus differentiating suicide from an accidental death or harm.

['Attempted suicide' is] "a potentially self injurious action with a non-fatal outcome for which there is evidence, either explicit or implicit, that the individual intended to kill himself or herself" (NI2016, p.107).

Again, while this may seem like a pragmatic, clarifying approach, by defining suicide as deliberate, the suicidal individual is positioned as the sole author of the suicidal act (Jaworski, 2015). The construction of suicide as deliberate, appears to cast suicide as a 
rational, cognitive act in a manner that makes emotional elements of suicidal distress somewhat invisible. In doing so, suicide can be understood as being positioned simplistically as the outcome of the agent's rational intentions, producing suicide as a primarily cognitive endeavour; meanwhile the suicidal individual as an emotional, feeling subject floats ghostlike and obscured behind dominant clinical, cognitive, and psychologised constructions of "suicidal thoughts" and "suicide behaviours".

As part of our commitment to the WPR approach, we analysed 'silences' in the policies, which are analytically important for drawing attention to salient elements that are ignored or forgotten (Bacchi and Goodwin, 2016). The present absence of the emotional suicidal individual is made starkly apparent when contrasted with the more emotional representations of, and engagements with, individuals bereaved by suicide.

"Losing a loved one to suicide is one is of life's most painful experiences. The feelings of loss, sadness, and loneliness experienced after any death of a loved one are often magnified in suicide survivors by feelings of guilt, confusion, rejection, shame, anger, and the effects of stigma and trauma" (NI2016, p.67).

The policies expressed concerns that individuals bereaved by suicide subsequently could be at heightened risk of suicide themselves, at times drawing on ideas of suicide contagion or clustering [1]. Uniquely among the policies, NI2012 included reflections from families bereaved by suicide. For example:

"A butterfly lights beside us like a sunbeam

And for a brief moment its glory and beauty belong to the world

But then it flies again

And though we wish it could have stayed

We feel lucky to have seen it" (NI2012, p.2).

In contrast to this, the emotional worlds of those experiencing suicidal distress were represented as somewhat more limited, primarily confined to brief references to suicidal persons feeling "hopeless", "helpless", "impulsive", "humiliated", "stressed" and "distressed" (NI2012\&2012; WAL2015). While the emotions of the bereaved were expanded on and explored, the emotions of those experiencing suicidal distress first-hand appeared to be conceptualised as reductionist risk factors for suicide, rather than complex parts of rich emotional lives which, for the purposes of suicide prevention, it could be important to understand.

The disconnection of suicide from emotions can be observed throughout suicide research more broadly, with suicide talked about in terms of suicidal thoughts and behaviours, with far less discussion of its emotional life (Jaworski, 2015). Where emotions are discussed within suicide research, it is often an abstract or reductionist manner (Chandler, 2019), that is characteristic of dominant psychologising ways of conceptualising emotions, reducing them to quantifiable measurements (Burkitt, 2014). Noticing the different depths of emotional engagement present within the policies led us to question how this difference had come about and what the consequences of it were. In this instance, we argue that this may be underlined by suicide stigma. In discussing the emotions of the bereaved, empathy 
is made possible between the policies' readers and those bereaved by suicide; allowing us to get inside the minds of the bereaved, by remembering or imagining what it is like to be bereaved by suicide.

In contrast, this opportunity for empathy is not afforded to suicidal individuals, perhaps motivated by a concern that in discussing the feelings of suicidal individuals we might expand our empathy too much, and that these feelings might be too dangerous to think about. However, as a consequence of this more limited engagement with the emotions of people who think about, attempt and die by suicide, an impoverished understanding of suicidal experiences is constructed, and the space in which empathy can be bridged between the reader and suicidal individuals is constrained. By primarily engaging with suicide as a deliberate act without considering the accompanying emotions, we construct a somewhat two-dimensional impression of the suicidal individual, obscuring the situated and embodied emotional lives of suicidal individuals, and in doing risk inadvertently othering individuals experiencing suicidal distress.

\section{Suicide as death-intentioned}

We have highlighted how suicide prevention policies construct suicide as individual, selfinflicted, and deliberate, preventable through a combination of surveillance, crisis intervention and medicalised, mental health care. This construction of suicide restricts suicide prevention opportunities to death prevention, whilst hiding suicidal people's emotions behind a curtain of pathologising language, positioning suicide as a rational, deliberate and intentioned act.

"Suicide is used in this document to mean a deliberate act that intentionally ends one's life" (ENG2012, p.5).

Whilst it is self-evident that the consideration of death is a necessary and integral part of suicide prevention, we propose advocating a suicide prevention approach that moves beyond the current focus on suicide as a single momentary act carried out by a pathologised, self-intentioned agent. Instead, we suggest that more attention could be focused on improving the material conditions for living as an equally valid approach to suicide prevention. Such an approach requires an explicit focus on contextual factors and structural changes which can make individual lives more liveable, positioning suicide within a landscape of broader political and economic inequalities (Ansloos, 2018; Standley, 2020).

To demonstrate the importance of enhancing the scope of these prevention policies, we can consider the 'priority people' identified within the policy documents. As noted above, these were groups such as unemployed people, LGBT people, asylum seekers, and people in contact with the criminal justice system. If we consider why these groups are at a heightened risk of suicide, it becomes impossible to ignore the social and material inequalities underlying this heightened prevalence and the structures which pattern such inequalities. However, it is not enough to simply acknowledge the complexity of suicide, we need to enhance our understandings of it, which requires a broader preventative focus than currently expressed in the policies we analysed. A re-conceptualization of suicide as socially situated and context dependent would encourage long-term commitment to questioning, 
disrupting, and transforming societal structures of power, in ways that may not be neatly quantifiable as directly preventing suicide. Enacting change in this area requires us to question the very norms and values around which our social and political systems are built, and the forms of inequality that they produce. Such a consideration would better enable the planning of initiatives and cross-departmental strategies to make changes to the material conditions under which lives become unliveable. The inherent limitations of the focus on quantifiable 'high-risk groups' is illustrated starkly in this note in the ENG12 policy:

In identifying the high-risk groups who are priorities for prevention (action 1), we have selected only those whose suicide rates can be monitored - this is essential if we are to report on what the strategy achieves (ENG12, pp.4).

We argue that by focusing on narrow, quantifiable prevention outcomes these documents ignore vital contextual factors. The quote above actively restricts its concern to groups whose deaths can be monitored. In doing so, it rules out groups whose suicide rates have not been nationally quantified, such as Black, Asian and Minority Ethnic people or lesbian, gay, bisexual and trans people, who may face structural violence and discrimination resulting in inequalities.

\section{Conclusion}

Throughout this article, we have argued that the suicidal individual invoked within the policy documents is somewhat two-dimensional: suicidal persons are the self that 'inflicts' death, dislocated from context, present in this action, but emotionally absent within the texts (East, Dorozenko and Martin, 2021). Perhaps as a consequence of this absent presence, suicidal individuals are primarily materialised in the texts in moments of life and death crisis; however, the conditions that lead up to the moment of a suicide attempt and the life trajectories of suicidal individuals following an interrupted, aborted, or survived suicide attempt remain largely unconsidered. The policies suggest improvements in the provision of mental health care subsequent to a suicide attempt through initiatives such as the 'zero suicide' ambition (in mental health care settings), psychosocial assessment for individuals who present in hospital with self-harm, and more empathetic attitudes to treatment from health care professionals. However, there remains a lack of discussion of the ways in which health services are, or are not, able to meet the needs of suicidal individuals in either quantity or quality of support provided, which should be of particular concern given the broad focus, both in the policy documents and within mental health campaigns, on encouraging those in distress to ask for help (Chandler, 2021).

By constructing suicide as primarily a mental health problem, crisis and mental health approaches to suicide prevention seek to offer neat, simple solutions, which are easily understood as directly focussing on suicide prevention (Hjelmeland and Knizek, 2017). This discursive move also mitigates responsibility because a pathological suicidal agent is not seen as responsible, but neither is government or society (Münster and Broz, 2015). The self-inflicted death thus becomes depoliticised, shielding the power structures that create conditions of unliveability (Butler, 2004). Suicide is therefore manoeuvred outside of the public and political sphere and into the private realm of individual pathology (Marsh, 2013; 
Rimke, 2016). In clinically focussed suicide research, it has been argued that psychologising approaches are privileged precisely because this is all a clinician can work with: the individual and their mind, due to a limited capacity to influence their individual client or patient's wider context. However, this is not the case in suicide prevention policies, which have the scope to potentially engage across a multitude of policy areas (Button, 2016; Yip and Tang, 2021). Therefore, we are left questioning what ends are served by focusing on the prevention of deaths by suicide through psychologised approaches, whilst choosing not to engage thoroughly across broader substantive policy areas to improve the material conditions that contribute to a life becoming unliveable (Ansloos, 2018).

In conclusion, we argue that attempts to narrowly define and conceptualise suicide can have significant consequences that impact whole suicide prevention strategies. Whilst defining suicide as self-inflicted, deliberate and death-intentioned could be seen as an attempt to eschew the possibility of accidental death or foul-play, in doing so suicide is curated as a private, individual death, dislocated from its broader context, and emotional life. As such, the 'problem' of suicide, whilst being superficially acknowledged as a complex biopsychosocial issue remains framed primarily as a mental health problem. Consequently, whilst some third sector initiatives were acknowledged as providing social support, these documents primarily offer clinically focussed mental health support and crisis intervention as a way of preventing death by suicide. This narrow focus on suicide as an individual mental health problem may have a constraining effect, resulting in suicide prevention policies restricting themselves to preventing the moment of death, at the expense of attempting to improve the material conditions for living. As a result, we suggest that these strategies do not realise their full potential to provide long term opportunities for suicide prevention, and we argue that equal consideration needs to be given to improving the structural and contextual conditions that can make life, for some, more liveable.

[1] Suicide contagion and clustering are terms used to describe relationships between individuals' suicides. Although these relationships are often ill-defined, it could be in terms of time, proximity, or methods (Cheng et al., 2014).

\section{References}

Ansloos, J. (2018) Rethinking Indigenous Suicide, International Journal of Indigenous Health, 13(2), pp. 8-28.

Bacchi, C. and Goodwin, S. (2016) Poststructural Policy Analyses - guide to practice. New York: Palgrave Macmillan.

Beutler, D. and Fenech, M. (2018) An analysis of the Australian Government's Jobs for Families Child Care Package: The utility of Bacchi's WPR methodology to identify potential influences on parents' childcare choices, Australasian Journal of Early Childhood, 43(1), pp. 16-24.

Braun, V. and Clarke, V. (2006) Using thematic analysis in psychology, Qualitative Research in Psychology, 3(2), pp. 77-101.

Burkitt, I. (2014) Emotions and Social Relations. London, Thousand Oaks, New Dehli, 
Singapore: SAGE Publications Ltd.

Butler, J. (2004) Undoing Gender. Oxfordshire: Routledge.

Button, M. E. (2016) Suicide and Social Justice: Toward a Political Approach to Suicide, Political Research Quarterly, 69(2), pp. 270-280.

Chandler, A. (2019) Socioeconomic inequalities of suicide: Sociological and psychological intersections, European Journal of Social Theory, pp. 1-19.

Chandler, A. (2021) Masculinities and suicide: unsettling "talk" as a response to suicide in men, Critical Public Health , pp. 1-10.

Cheng, Q, Li H, Silenzio, V, Caine ED. (2014) Suicide contagion: A systematic review of definitions and research utility, PLoS ONE. Vol. 26; 9(9):e108724.

East, L., Dorozenko, K. P. and Martin, R. (2021) The construction of people in suicide prevention documents, Death Studies, 45(3), pp. 182-190.

Fitzpatrick, S. J. (2021) The moral and political economy of suicide prevention, Journal of Sociology, pp. 1-17.

Fitzpatrick, S. J., Hooker, C. and Kerridge, I. (2015) Suicidology as a social practice,, Social Epistemology, 29(3), pp. 303-322.

Hjelmeland, H. and Knizek, B. L. (2017) Suicide and mental disorders: A discourse of politics, power, and vested interests, Death Studies, 41(8), pp. 481-492.

Jaworski, K. (2015) Suicide, Agency and the Limits of Power, in Broz, L. and Munster, D. (eds) Suicide and Agency: Anthropological Perspectives on Self-Destruction, Personhood, and Power. Farnham: Routledge, pp. 183-201.

Lampropoulos, D. and Apostolidis, T. (2021) Representing the citizenship of mental health users in French mental health policy: A critical analysis of the official French texts on mental health policies since 2005, Critical Social Policy, 41(1), pp. 25-45.

Lancaster, K., Rhodes, T. and Rance, J. (2020) "Towards eliminating viral hepatitis":

Examining the productive capacity and constitutive effect o global policy on hepatitis $C$ elimination, International Journal of Drug Policy, 80, pp. 1-8.

Mackley, B. A. (2019) Suicide Prevention : Policy and Strategy, House of commons library. Available at: https://commonslibrary.parliament.uk/research-briefings/cbp-8221/.

Accessed: 18 December 2020

Marsh, I. (2013) The uses of history in the unmaking of modern suicide, Journal of Social History, 46(3), pp. 744-756.

Marsh, I. (2016) Critiquing Contemporary Suicidology, in Critical Suicidology. Transforming Suicide Research and Prevention for the $21^{\text {st }}$ Century, pp. 15-30.

Mills, C. (2018) "Dead people don't claim": A psychopolitical autopsy of UK austerity suicide, Critical Social Policy, 38(2), pp. 302-322.

Münster, D. and Broz, L. (2015) The anthroppology of suicide: Ethnography and the tension of agency, Suicide and Agency: Anthropological Perspectives on Self-Destruction, 
Personhood and Power, pp. 3-23.

Northern Ireland Assessmbly Research and Information Service (2019) Suicide statistics and strategy in Northern Ireland: Update - Research Matters. Available at:

https://www.assemblyresearchmatters.org/2019/11/28/suicide-statistics-and-strategy-innorthern-ireland-update/ (Accessed: 29 April 2021).

Office for National Statistics (2019) Suicides in the UK: 2018 registrations. Available at: https://www.ons.gov.uk/peoplepopulationandcommunity/birthsdeathsandmarriages/death s/bulletins/suicidesintheunitedkingdom/2018registrations\#suicides-by-country (Accessed: 29 April 2021).

Rimke, H. (2016) Introduction - Mental and Emotional Distress as a Social Justice Issue: Beyond Psychocentrism, Studies in Social Justice, 10(1), pp. 4-17.

Silverman, M. M. (2016) Challenge to Defining and Classifying Suicide and Suicidal Behaviours, in in O'Connor, R. C. and Pirkis, J. (eds) The International Handbook of Suicide Prevention. Second Edi. Wiley Blackwell, pp. 11-35.

Standley, C. J. (2020) Expanding our paradigms: Intersectional and socioecological approaches to suicide prevention, Death Studies. pp. 1-9.

Stewart, M. (2019) Preventable harm: creating a mental health crisis, Journal of Public Mental Health, 18(4), pp. 224-230.

Stewart, M. (2020) What price preventable harm: social policies designed to disregard human need, The Centre for Welfare Reform, pp. 1-13.

White, J. (2017) What can critical suicidology do?, Death Studies, 41(8), pp. 472-480.

World Health Organization (2021) LIVE LIFE. An implementation guide for suicide prevention in countries. Available at: https://apps.who.int/iris/rest/bitstreams/1350957/retrieve. Accessed: 7 March 2018.

Yip, P. S. F. (2005) A public health approach to suicide prevention, Hong Kong Journal of Pscyhiatry, 15(1), pp. 29-31.

Yip, P. S. F. and Tang, L. (2021) Public Health Approach to Suicide Research, in Kolves, K. et al. (eds) Adavancing Suicide Research. Boston; Gottingen: Hogrefe Publishing, pp. 29-38. 